\title{
Stable Skyrmions in spinor condensates
}

\author{
Igor F. Herbut ${ }^{1,2}$ and Masaki Oshikawa ${ }^{2}$ \\ 1 Department of Physics, Simon Fraser University, Burnaby, British Columbia, Canada V5A $1 S 6$ \\ 2 Department of Physics, Tokyo Institute of Technology, \\ Oh-okayama, Meguro-ku, Tokyo 152-8551, Japan
}

\begin{abstract}
Globally symmetric spinor condensates in free space are argued not to support stable topological defects in either two or three dimensions. In the latter case, however, we show that a topological Skyrmion can be stabilized by forcing it to adopt certain density profiles. A sufficient condition for the existence of Skyrmion solutions in three dimensions is formulated and illustrated in simple examples. Our results pertain to Bose-Einstein condensation in ${ }^{87} R b$.
\end{abstract}

Topologically non-trivial configurations play a crucial role in our present understanding of ordering, dynamics, and criticality in basic models of statistical mechanics [1]. Probably the best understood example is that of vortices and vortex-loops in the Ginzburg-Landau, or Higgs, $O(2)$-symmetric theory for a complex order parameter in two $(2 \mathrm{D})$ and three dimensions $(3 \mathrm{D})$, which are responsible for the very existence of the disordered phase at high temperatures [2]. The next in order of increasing complexity is the $O(3)$-symmetric Heisenberg model, which allows texture-like Skyrmions in 2D [3] , and point-like hedgehogs in 3D [4]. Whereas Skyrmions in 2D are understood to provide an additional source of disorder for the low-temperature phase, the exact role of hedgehogs in the 3D Heisenberg model is already less clear [5]. Both, nevertheless, represent non-trivial spatial configurations, which by virtue of their distinct topology are locally stable and separated from the ground state by an infinite energy barrier.

In this Letter we study the issue of topological defects in the next simplest case: the order parameter with global $O(4)$ symmetry. Such a symmetry arises in complex condensates with an internal spin-1/2-like quantum number [6], for example. Realizations of such spinor condensates are found in models of inflatory cosmology 7] , Bose-Einstein condensation of ${ }^{87} R b[\underline{8}]$, bosonic ferromagnetism [6], 9], and in effective theories of hightemperature superconductivity [10], 11], and of deconfined criticality [12. The Higgs sector of the WeinbergSalam model of electro-weak interactions represents another closely related example, with a spinor condensate coupled to gauge fields. It is easy to show that the triviality of the first and the second homotopy groups of the three-dimensional unit sphere, $S_{3}$, on which the order parameter lives at low temperatures, implies that there are no stable topological defects in two dimensions. 2D spinor condensates may therefore be expected to be in the disordered phase at all finite temperatures, precisely as described by the $O(4)$ non-linear $\sigma$-model $(N L \sigma M)$. In $3 \mathrm{D}$, on the other hand, standard topological considerations suggest the possibility of a Skyrmion texture, thanks to the third homotopy group of $S_{3}$ being the group of integers. Here, however, unexpected subtleties arise, which together with the growing relevance of the problem provide the motivation for the present work.
It has been known that finite-energy textures in higher than two dimensions are generally unstable with respect to shrinkage 13. Derrick's scaling argument, does not, however, forbid topological defects with infinite energy, hedgehogs in the 3D Heisenberg model being a prime example. The search for stable topological defects in the $O$ (4) $N L \sigma M$ in $3 \mathrm{D}$, however, has so far led to a negative result [14], 15], 16], 17]. We first demonstrate that even with amplitude variations included the spherically symmetric Skyrmion remains unstable. This conclusion follows from a useful mechanical interpretation of Skyrmion's differential equation, in which the radial dependence of particle's density appears as a 'source of dissipation' for a fictitious classical particle, which prevents it from oscillating with a full amplitude. This hindrance, however, may be turned into an advantage by forcing the same radial dependence to have a form that effectively serves as an 'energy pump'. This idea yields an integral condition on the density profile of a Skyrmion, which we illustrate with three qualitatively different analytical solutions of the non-linear Euler-Lagrange's equations. Skyrmion solutions are found, for example, for specific forms of the confining potential, which may be used in creating such configurations in the laboratory [8].

Let us begin by defining the system of two-component bosons in the continuum with the standard action in terms of the complex coherent states written as

$$
S=\int_{0}^{\beta} d \tau d^{3} \vec{x}\left[\Phi^{\dagger}\left(\partial_{\tau}-\nabla^{2}-\mu+V(\vec{x})\right) \Phi+\frac{U}{2}\left(\Phi^{\dagger} \Phi\right)^{2}\right]
$$

where $\Phi^{\dagger}=\left(\Phi_{1}^{*}(\vec{x}, \tau), \Phi_{2}^{*}(\vec{x}, \tau)\right)$. We have also included a confining potential $V(\vec{x})$, assumed a contact repulsion $U$ for simplicity, and set $\hbar=1$, and the boson mass $2 m=1$. $\beta=1 / k_{B} T$ is the inverse temperature.

We are interested in finding classical, that is $\tau$ independent, locally stable field configurations of the action (1). The field $\Phi(\vec{x})$ may be written in terms of its real amplitude $f(\vec{x})$ and the normalized complex spinor $a(\vec{x})$ as $\Phi^{\dagger}=f a^{\dagger}$, with $a^{\dagger} a=1$. The low-energy physics is then described by fluctuations of the spinor $a$, which can be identified with a four-component real vector of unit length. The symmetry of the order parameter is thus $O(4)$. Varying the above action leads to the requisite Euler-Lagrange's equations

$$
f\left(a^{\dagger} \nabla^{2} a+\left(\nabla^{2} a^{\dagger}\right) a\right) a=2 f \nabla^{2} a+4(\nabla f) \cdot(\nabla a),
$$




$$
-\nabla^{2} f+\left[\left(\nabla a^{\dagger}\right) \cdot(\nabla a)-1+V\right] f+f^{3}=0,
$$

where we have rescaled the lengths as $\vec{x} \sqrt{\mu} \rightarrow \vec{x}$, the amplitude as $f \sqrt{U / \mu} \rightarrow f$, and the external potential as $V / \mu \rightarrow V$. Solution needs to satisfy the boundary conditions

$$
\lim _{x \rightarrow \infty} x^{2} f^{2} \nabla a=0
$$

which guarantee stability with respect to small rotations of the spinor $a$ at the infinitely remote boundary of the system.

Eqs. (2) and (3) form a set of five differential equations for real and imaginary parts of the spinor $a$ and the amplitude $f$. In a spherically symmetric potential $V(\vec{x})$ we can partially solve them, however, by assuming the most general ansatz with the same symmetry $[4]: f(\vec{x})=f(r)$,

$$
a^{\dagger}(\vec{x})=\left(\sin \omega(r) \cos \theta+i \cos \omega(r), \sin \omega(r) \sin \theta e^{i \phi}\right),
$$

where $\vec{x}=(r \sin \theta \cos \phi, r \sin \theta \sin \phi, r \cos \theta)$. Somewhat tedious but otherwise straightforward algebra shows that all four of Eqs. (2) will then be satisfied provided that the function $\omega(r)$ satisfies the differential equation

$$
\frac{d^{2} \omega(r)}{d r^{2}}+\left[\frac{2}{r}+\frac{2}{f(r)} \frac{d f(r)}{d r}\right] \frac{d \omega(r)}{d r}-\frac{\sin (2 \omega(r))}{r^{2}}=0
$$

Similarly, Eq. (3) then reduces to

$$
\begin{array}{r}
-\frac{d^{2} f(r)}{d r^{2}}-\frac{2}{r} \frac{d f(r)}{d r}+\left[\left(\frac{d \omega(r)}{d r}\right)^{2}+\right. \\
\left.2\left(\frac{\sin \omega(r)}{r}\right)^{2}-1+V(r)\right] f(r)+f^{3}(r)=0 .
\end{array}
$$

The action for the ansatz (5) is

$$
\begin{aligned}
& \frac{S}{4 \pi \beta}=\int_{0}^{\infty} r^{2}\left[\left(\frac{d f}{d r}\right)^{2}+\left(\left(\frac{d \omega}{d r}\right)^{2}+\right.\right. \\
& \left.\left.2\left(\frac{\sin \omega}{r}\right)^{2}+V(r)-1\right) f^{2}+\frac{f^{4}}{2}\right] d r,
\end{aligned}
$$

so that Eqs. (6) and (7) may also be recognized as EulerLagrange's equations for the latter form of $S$.

The ansatz for $a(\vec{x})$ will be topologically non-trivial and wrap the $S_{3}$, defined by the condition $a^{\dagger} a=1, N$ times if, as the radius varies from zero to infinity, the function $\omega(r)$ takes all the values from zero to $N \pi$. Hereafter we restrict our discussion only to the elementary Skyrmion with $N=1$.

If we turn the external potential off $(V(r) \equiv 0)$, it is easy to show that the Eqs. (6) and (7) do not actually have the Skyrmion as a solution, the assumptions to the contrary notwithstanding [15], 16]. To this purpose define a new 'time' variable $-\infty<t=\ln r<\infty$. Eq. (6) then becomes

$$
\ddot{\omega}(t)=-\frac{d W(\omega)}{d \omega}-\eta(t) \dot{\omega}(t),
$$

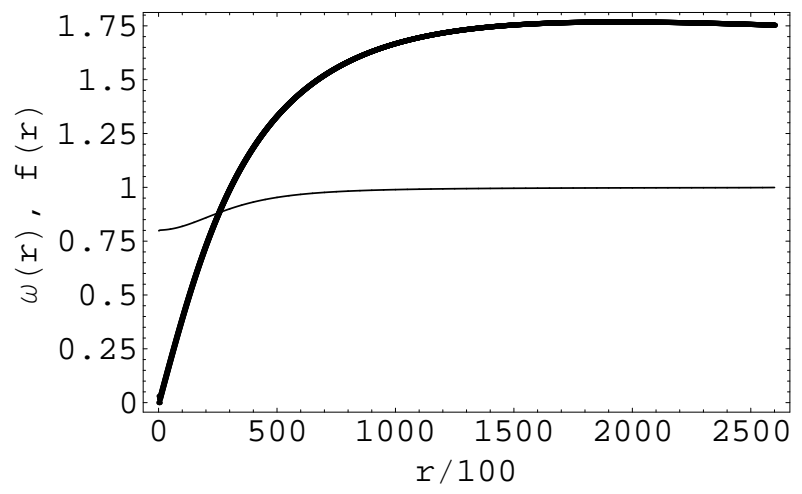

FIG. 1: A numerical solution of Eqs. (6) and (7): $\omega(r)$ (thicker curve) and $f(r)$.

with $W(\omega)=(\cos 2 \omega) / 2, \eta(t)=1+d \ln f^{2}(t) / d t$, and $\dot{\omega}=d \omega / d t$. The function $\omega(t)$ may now be interpreted as a coordinate of a particle moving in the potential $W(\omega)$, coupled to a dissipative environment with the time-dependent coefficient of dissipation $\eta(t)$. Let us first neglect the amplitude variations dictated by Eq. (7). Dissipation then still persists, and $\eta(t) \equiv 1$. Demanding that $\omega(0)=0$ Eq. (6) implies $\omega(r) \sim r$ for $r \ll 1$, so that the initial condition in our mechanical analogy is $\omega(-\infty)=\dot{\omega}(-\infty)=0$. This corresponds to rolling down from the top of the potential $W(\omega)$ without any initial kinetic energy. Independently of the slope of $\omega(r)$ at the origin the solution therefore always oscillates around and approaches $\omega(\infty)=\pi / 2$, dissipating energy and loosing its amplitude in the process. Eq. (6) for a fixed amplitude therefore admits only a meron (halfSkyrmion) as a solution. However, since when $r \gg 1$, $|\omega(r)-\pi / 2| \sim 1 / \sqrt{r}$ for the meron, the boundary condition in Eq. (4) is not satisfied. Consequently, the meron is unstable with respect to deformation $\omega(r) \rightarrow \omega(r)-\epsilon(r)$ with $\epsilon(0)=0$ and $0<\epsilon(\infty) \ll 1[18$.

Before relaxing the condition of fixed amplitude let us observe that solutions of Eq. (6) (still with $f(r)=$ const) that satisfy the boundary conditions (4) and approach $\omega(\infty)=\pi$ as $\omega(r) \sim \pi \pm 1 / r^{2}$, do in fact exist. These solutions, however, are singular near the origin, $\omega(r) \sim$ $\pm 1 / r$, and hence have an infinite action. We will refer to these as singular solutions and discuss their possible significance in a moment.

Next, we retain $V(r) \equiv 0$, but allow the amplitude variations. Finiteness of the action, measured from the value for the ground state $f(r) \equiv 1, \omega(r)=0$, requires then $f(\infty)=1$. Linearizing near the origin one still finds $\omega(r)=A r+O\left(r^{2}\right)$, and $f(r)=f(0)+(f(0) / 6)\left(f^{2}(0)+\right.$ $\left.3 A^{2}-1\right) r^{2}+O\left(r^{3}\right)$, where $A$ and $f(0)$ are finite constants. To have $f(\infty)=1$ we then find numerically that $f(0)<1$, and $f(r)$ to be a weakly and uniformly increasing function (Figure 1). The reason behind such a behavior is obvious: gradients of $a(\vec{x})$ are largest near the origin, so suppressing the amplitude there somewhat always lowers the energy. Energy density being integrable, 
however, the amplitude remains finite at the origin, and the Skyrmion is 'coreless'. With such a solution for $f(r)$, however, one still finds $\omega(\infty)=\pi / 2$ and Eq. (4) violated. This is evident from our mechanical analogy in Eq. (9) in which having the amplitude increasing with radius only adds to 'dissipation'.

The stage is now set for the main part of the Letter. The preceding analysis points to a simple way to stabilize the Skyrmion. To have a solution of Eqs. (6) and (7) satisfying the requisite boundary conditions $\omega(t=-\infty)=\dot{\omega}(t=-\infty)=0$, and $\omega(t=\infty)=\pi$, $\dot{\omega}(t=\infty)=0$, it is necessary and sufficient that the total dissipation in our mechanical analogy vanishes. Mathematically [19]:

$$
\int_{-\infty}^{\infty} \eta(t) \dot{\omega}^{2}(t) d t=0
$$

The total mechanical, and thus the potential, energy is then the same at the initial and the final 'time' $t$. The amplitude therefore must be a decreasing function at least for some radia, where it would 'pump' the energy back into the oscillator. This is our main result. In the remaining we provide some specific amplitude profiles that indeed lead to stable Skyrmions.

1) The trivial case is the one without dissipation: $\eta(t) \equiv 0$. This is equivalent to the particle density

$$
f^{2}(r)=\frac{A}{r},
$$

with $A$ as a constant. This yields

$$
\omega(r)=2 \cot ^{-1}\left(r^{\sqrt{2}}\right) .
$$

Inserting this solution into Eq. (7) gives the external potential that enforces such a density to be

$$
V(r)=1-\frac{A}{r}-\frac{1}{4 r^{2}}-\frac{16 r^{2(\sqrt{2}-1)}}{\left(1+r^{2 \sqrt{2}}\right)^{2}} .
$$

It is easy to check that this solution has a finite action, due to the singularity of the external potential at the origin. The same singularity, however, makes this simplest example less than completely satisfying from a possible practical point of view.

2) The difficulty in utilizing the condition in Eq. (10) is that the velocity $\dot{\omega}(t)$ of course depends on the dissipation $\eta(t)$ in a rather non-trivial way, so it appears that solving the differential equations (6) and (7) is always unavoidable in practice. This, fortunately, is not so, due to the following theorem: For potentials $W(\omega)$ that are even functions of $\omega-\pi / 2$, and dissipation coefficients $\eta(t)$ that are odd functions of $t$, solutions of Eq. (9) are such that

$$
\omega(t)-\frac{\pi}{2}=-\omega(-t)+\frac{\pi}{2},
$$

i.e. odd functions of time, measured from the moment when at the bottom of the potential. It is thus sufficient to have a density profile that implies an odd $\eta(t)$ to

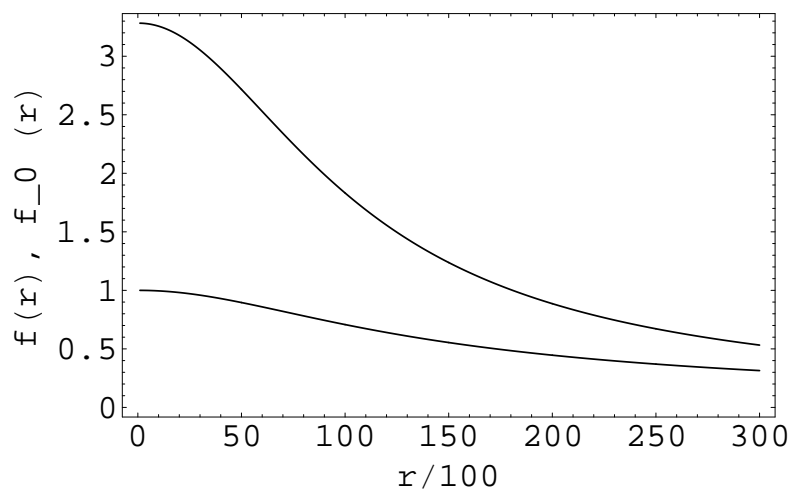

FIG. 2: Topologically trivial ground state $f_{0}(r)$ (upper curve) and the Skyrmion's amplitude $f(r)$, in the potential in Eq. (18) with $B=1$.

stabilize the Skyrmion. As an illustration, consider

$$
\eta(t)=-\tanh (t),
$$

for which the the particle density is simply

$$
f^{2}(r)=\frac{B}{1+r^{2}},
$$

with $B$ as a constant. It is straightforward to check that the solution of Eq. (7) is then

$$
\omega(r)=2 \cot ^{-1}(r),
$$

and the corresponding external potential

$$
V(r)=1-\frac{B}{1+r^{2}}-\frac{15}{\left(1+r^{2}\right)^{2}} .
$$

Different density profiles can be similarly constructed, including those that would lead to a finite total number of particles.

We may note that for the external potential in Eq. (18) there is yet another, topologically trivial, solution of Eqs. (6) and (7): $\omega(r)=0, f_{0}(r)$. Numerical solution for $f_{0}(r)$ for $B=1$ is plotted on Figure 2. Since $f_{0}(r)>f(r)$, the action, which at a stationary point takes the form

$$
\frac{S}{4 \pi \beta}=-\frac{1}{2} \int_{0}^{\infty} r^{2} f^{4}(r) d r,
$$

is actually lower for $f_{0}(r)$. This represents the ground state configuration in the potential (18).

3) Finally, the Skyrmion may be stable in a constant, but discontinuous, particle density as well. Take $f^{2}(r)=$ $e^{C}$ for $r<1$, and $f^{2}(r)=1$ for $r>1$, for example. Then

$$
\frac{d \ln f^{2}(t)}{d t}=-C \delta(t),
$$

and the total dissipation will vanish for $C$ tuned to

$$
C=\int_{-\infty}^{\infty}\left[\frac{\dot{\omega}(t)}{\dot{\omega}(0)}\right]^{2} d t
$$


Such a discontinuity in the amplitude may be understood as providing a 'kick' in our mechanical terminology, which instantaneously increases the kinetic energy at $t=0$ so that there is just enough to reach the next top of the potential $W(\omega)$ at $\omega=\pi$. This is possible because for any regular solution of Eq. (6) (with $f=$ const) which starts at $\omega(r=0)=0$ there is a singular solution that intersects it at $r=1$, and which asymptotically approaches $\pi$ as the radius increases. Their difference in slopes at $r=1$ determines the required injection of energy measured by the discontinuity $C$.

Previously, the Skyrmion was found to be stable numerically if the $O(4)$ symmetry of the action in Eq. (1) is broken down to $O(2) \times O(2)$, in a way that favors phase separation [17]. In contrast, our action is fully $O(4)$ symmetric, and the stability obtains from the interplay between the form of the Skyrmion and its density profile. Derrick's scaling argument is likewise evaded by the introduction of the preferred length scale for density variation. We hope our results will help facilitate the production of these interesting topological objects in laboratory spinor condensates, such as ${ }^{87} R b$. Recent advances in (dynamic) manipulation of confining potentials seem particularly promising in this respect [20].

Finally, for completeness let us revisit the issue of defect's stability in 2D. Consider

$$
a^{\dagger}(\vec{x})=\left(\sin \lambda \cos \theta(r)+i \cos \lambda, \sin \lambda \sin \theta(r) e^{i \phi}\right),
$$

where $\vec{x}=r(\cos \phi, \sin \phi)$, and $\theta(0)=0$ and $\theta(\infty)=$ $\pi / 2$. For a fixed parameter $\lambda=\pi / 2$ this reduces to the configuration often discussed in literature [8]. It is evident, however, that by tuning $\lambda$ one may continuously deform the defect at $\lambda=\pi / 2$ to the trivial vacuum at $\lambda=0$, monotonically decreasing the action along the way. This construction exploits the fact that $\pi_{2}\left(S_{3}\right)=$ 1. Topological defects in spinor condensates are thus in general unstable in free space both in $2 \mathrm{D}$ and in $3 \mathrm{D}$, although for rather different reasons.

This work has been supported by NSERC of Canada (IFH), Grant-in-Aid for Scientific Research (MO), and 21st Century COE Program at Tokyo Institute of Technology, 'Nanometer scale Quantum Physics' (IFH and MO), from MEXT of Japan. The authors also acknowledge helpful conversations with K. Ino, M. Kennett, J. McGuirk, B. Seradjeh, and M. Ueda.
[1] P. M. Chaikin and T. C. Lubensky, Principles of Condensed Matter Physics, (Cambridge University Press, 1995).

[2] I. Herbut, A Modern approach to Critical Phenomena, (Cambridge University Press, 2006), Chapters 6 and 7.

[3] A. A. Belavin and A. M. Polyakov, JETP Lett. 22, 245 (1975).

[4] N. Manton and P. Sutcliffe, Topological Solitons, (Cambridge University Press, 2004).

[5] M. Lau and C. Dasgupta, Phys. Rev. B 39, 7212 (1989); K. Kamal and G. Murthy, Phys. Rev. Lett. 71, 1911 (1993); N. D. Antunes, L. M. A. Bettencourt, and M. Kunz, Phys. Rev. E 65, 066117 (2002); O. I. Motrunich and A. Vishwanath, Phys. Rev. B 70075104 (2004).

[6] E. Eisenberg and E. H. Lieb, Phys. Rev. Lett. 89, 220403 (2002).

[7] A. Vilenkin and A. P. S. Shellard, Cosmic Strings and Other Topological Defects, (Cambridge University Press, 1994).

[8] For a recent review see, K. Kasamatsu, M. Tsubota, and M. Ueda, Int. J. of Mod. Phys. B, 19, 1835 (2005).

[9] Y. Saiga and M. Oshikawa, Phys. Rev. Lett. 96, 036406 (2006).

[10] P. A. Lee, N. Nagaosa, T.-K. Ng, and X.-G Wen, Phys.
Rev. B, 57, 6003 (1998).

[11] I. F. Herbut, Phys. Rev. Lett. 94, 237001 (2005); ibid. 88, 047006 (2002); Phys. Rev. B 66, 094504 (2002).

[12] T. Senthil and M. P. A. Fisher, Phys. Rev. B 74, 064405 (2006).

[13] G. H. Derrick, J. Math. Phys. 51252 (1964).

[14] L. Lichtensteiger and R. Durrer, Phys. Rev. D 59, 125007 (1999).

[15] U. Al Khawaja and H. T. C. Stoof, Nature (London) 411, 918 (2001); Phys. Rev. A 64, 043612 (2001).

[16] J. Ruostekoski and J. R. Anglin, Phys. Rev. Lett. 86, 3934 (2001).

[17] See also, R. A. Battay, N. R. Cooper, and P. M. Sutcliffe, Phys. Rev. Lett. 88, 080401 (2002) for a (numerically) stable skyrmion in the theory with a reduced symmetry.

[18] In contrast, in the $O(3) N L \sigma M$ in $2 \mathrm{D}$ the parametrization $\vec{n}=(\sin \omega(r) \cos \phi, \sin \omega(r) \sin \phi, \cos \phi)$ leads to a similar equation for $\omega(t)$, but with $\eta(t) \equiv 0$, facilitating this way the usual Belavin-Polyakov Skyrmion.

[19] L. D. Landau and E. M. Lifshitz, Mechanics, (Oxford, England: Pergamon, 1976), Ch. 5.

[20] V. Boyer, et. al, Phys. Rev. A 73, 031402 (2006). 